\title{
5 Transparenz und Public Reporting
}

\subsection{Begriffsbestimmung und gesetzliche Grundlagen}

Public Reporting oder Public Disclosure beschreibt die Veröffentlichung von Qualitätsdaten (SVR 2008 Nr. 698), die mittels definierter Indikatoren gemessen werden. Die geringste Anforderung an die Definition von Public Reporting besteht in einer lediglich internen Deanonymisierung, die Leistungserbringer kennen also selbst ihre Daten, verfügen ansonsten aber nur über eine anonymisierte Information über die Verteilung, und die Öffentlichkeit kennt nur anonymisierte Daten (sog. Private Disclosure, man kann diese Form auch aus der Definition von Public Reporting ausschließen). Damit die Informationen ihre Wettbewerbswirksamkeit jedoch voll entfalten können, müssen sie auch bezüglich Institution oder behandelndem Arzt für die Öffentlichkeit deanonymisiert sein (Public Disclosure). In einzelnen Fällen wird sogar vorgeschlagen, einzelne unerwünschte Ereignisse unter Nennung von Institution und Behandler zu veröffentlichen. In den USA wird teilweise neben der Qualitätsinformation auch eine Kosteninformation gegeben, damit die Patienten eine Entscheidung bezüglich des value of care treffen können (Hibbard et al. 2012).

Im Kontext des Begriffes des Qualitätswettbewerbs und in Abgrenzung zu Pay for Performance ist weiterhin festzuhalten, dass Public Reporting durchaus über ökonomische Anreize wirkt, aber in indirekter Form. Qualitätsinformationen werden nicht finanziell bewertet und vergütet, sondern es besteht die Absicht, das „Kundenverhalten“ in dem Sinne zu beeinflussen, dass die „besseren“ 
Einrichtungen mehr Patienten haben und daher im Markt besser bestehen können (s. Wasem und Geraedts 2011). Als „Kunden“ sind hier einerseits die Zuweiser und Patienten anzusehen (sog. Behandlungsmarkt), andererseits die Krankenkassen, die ebenfalls Kenntnis von den Qualitätsinformationen haben und dies in die Verhandlungen mit den Leistungsanbietern einbringen. Von allen drei Gruppen (Patienten, Zuweiser, Versicherungen) wird angenommen, dass sie sich für diese Informationen interessieren, sich diese beschaffen, diese verstehen und sich danach verhalten, ein weites Feld, wie sich in der weiteren Analyse noch zeigen wird (s. Kap. 5.3). Es wird weiterhin richtigerweise darauf hingewiesen, dass die Einrichtungen letztendlich auch selber die Qualitätsinformationen nutzen können, indem sie diese z.B. für interne Verbesserungsprozesse nutzen oder sie in die eigene Marktpositionierung einbringen (s. Wasem und Geraedts 2011). Handelt es sich lediglich um Private Disclosure, haben die externen Partner nur die Möglichkeit, auf das Vorhandensein dieser Daten zu verweisen und das (offene oder nicht offene) Umgehen mit diesen Daten in ihren Entscheidungen zu bewerten.

Zusammenfassend kann auf dieser Basis heute von folgender Definition von Public Reporting ausgegangen werden:

In Deutschland ist Public Reporting zunächst mit lediglich institutionsinterner Veröffentlichung der Qualitätsdaten seit der GKV-20oo Reform vom 17.12.1999 im Sinne der Verpflichtung, „sich an einrichtungsübergreifenden Maßnahmen der Qualitätssicherung zu beteiligen, die insbesondere das Ziel haben, die Ergebnisqualität zu verbessern“ ( $\$ 135$ a Abs. 2), im Gespräch. Die weitere Entwicklung führte zu den jährlichen Berichten der Bundesgeschäftsstelle Qualitätssicherung und zur Verpflichtung im Fallpauschalengesetz vom 14.12.2001, alle zwei Jahre (später jährlich) einen Qualitätsbericht zu veröffentlichen ( $\$ 137$ Abs. 1 Satz 3 Nr. 6). Es dauerte bis zum GKV-Wettbewerbsstärkungsgesetz vom 26.3.2007, dass daraus ein Public Reporting Ansatz entstand, mit dem auch die externe Öffentlichkeit die Qualitätsdaten der jeweiligen Institution zuzuordnen konnte. Dies war Aufgabe der „fachlich unabhängigen Institution“, die mit dem neu eingefügten $₫ 137$ a SGB V beauftragt wurde, ,für die Messung und Darstellung der Versorgungsqualität möglichst sektorenübergreifend abgestimmte Indikatoren und Instrumente zu entwickeln" sowie „die Ergebnisse ... durch die Institution in geeigneter Weise und in einer für die Allgemeinheit verständlichen Form zu veröffentlichen“ ( $\$$ 137a Abs. Nr. 1 und 4). Diese Institution, deren Funktion durch das AQUA-Institut übernommen wurde, stand unter der Richtlinienhohheit des Gemeinsamen Bundesausschusses, der nach $\mathbb{\$} 137$ die Inhalte des Qualitätsberichtes bestimmen muss, der „von den Landesverbänden der Krankenkassen und den Ersatzkassen im Internet zu veröffentlichen“ ist ( $\$ 137 \mathrm{Abs}$. 3 Nr. 4). Im Qualitätsreport 2012 standen 289 Indikatoren, davon 74 risikoadjustiert, zur Veröffentlichung an (AQUA 2013). 
Public Reporting (PR) im Gesundheitswesen stellt Qualitätsinformationen in einer Form zur Verfügung, in der sie das Verhalten der externen Partner einer Gesundheitseinrichtung beeinflussen (Patienten, Zuweiser, Versicherungen). Sie basiert auf einer Qualitätsmessung durch definierte Qualitäts- oder Patientensicherheitsindikatoren und stellt diese Information deanonymisiert zur Verfügung, entweder nur gegenüber der internen (institutionellen) oder gegenüber der externen Öffentlichkeit (mit Nennung von Institution und/oder Arzt). Möglich ist weiterhin die Nennung von einzelnen Zwischenfällen mit allen Verantwortlichen. Zur Darlegung der Effizienz (value of care) kann neben den Qualitätsindikatoren auch über die Behandlungskosten informiert werden.

Die in Kapitel 2.1 geäußerte Kritik an der Zusammensetzung der Qualitätsindikatoren und ihrer Überbetonung der operativen (Maximal-)Versorgung soll nicht darüber hinwegtäuschen, dass die geschilderte Entwicklung in nur etwas mehr als 10 Jahren einen erheblichen Schritt in die richtige Richtung darstellt. Die technische Auswertung und die Darstellung der Indikatoren in den Qualitätsberichten von BQS (bis 2008) und AQUA-Institut stellen hervorragende Quellen für (die beschriebenen) Qualitätsdaten dar und sind auch im internationalen Rahmen beachtenswert.

Am ehesten kann man die Entwicklung in den USA als Analog heranziehen (vgl. Darstellung in Kap. 4.5). Im Rahmen einer sehr umfassenden Diskussion und Konzeptbildung zur Qualitätsproblematik wurde 2003 im Medicare Prescription Drug, Improvement and Modernization Act (MMA) und 2005 im Deficit Reduction Act ein Public Reporting-Programm eingeführt (IOM 2007, CMS 2012, weiterhin Belmont et al. 2011, Corrigan und McNeill 2009, Luft 2012). Es werden Indikatorensets zu insgesamt neun Bereichen der Gesundheitsversorgung publiziert, u.a. aus der stationären Krankenhausversorgung (Hospital Inpatient Quality Reporting Program [Hospital-IQR]), der ambulanten Krankenhausversorgung, aus Arztpraxen, der stationären und häuslichen Pflege und den Krankenkassen bzw. Managed Care Organisationen (Medicare C und D, s. CMS 2012A). Einer der Unterschiede zum deutschen System besteht darin, dass Patientenbefragungen bzw. Patient-Reported Outcome Measures eine große Bedeutung zukommt (z.B. 11 Indikatoren beim Hospital-IQR). 90\% der anderen Indikatoren sind Prozessindikatoren und werden nicht risikoadjustiert.

\section{Zusammenfassung:}

Public Reporting beruht auf indirekten ökonomischen Anreizen, die von öffentlich zugänglichen Qualitätsinformationen ausgehen. Public Reporting basiert auf einer Qualitätsmessung durch definierte Qualitäts- oder Patientensicherheitsindikatoren und stellt diese Information deanonymisiert zur Verfügung, 
entweder nur gegenüber der internen (institutionellen) oder gegenüber der externen Öffentlichkeit (mit Nennung von Institution und/oder Arzt). Möglich ist weiterhin die Nennung von einzelnen Zwischenfällen mit allen Verantwortlichen. Zur Darlegung der Effizienz (value of care) kann neben den Qualitätsindikatoren auch über die Behandlungskosten informiert werden.

\subsection{Wirksamkeit: differenzierte Betrachtung notwendig}

Die nicht-anonymisierte Veröffentlichung von Qualitätsdaten (public disclosure) erzeugt die Anreizwirkung insbesondere über einen befürchteten Reputationsverlust. Die wissenschaftlichen Studien zu den Auswirkungen auf die Qualität der Versorgung ergeben auf den ersten Blick ein unübersichtliches und widersprüchliches Bild (s. frühe Metaanalyse von Marshall et al. 2000). In dem vom Sachverständigenrat in seinem Gutachten von 2007 publizierten Analyse (SVR 2008 Nr. 695ff) wurden die Studien hinsichtlich der untersuchten Adressaten der Qualitätsverbesserung (Patienten, Einweiser, Leistungserbringer) und hinsichtlich der Endpunkte (Verbesserung von Prozess- und Ergebnisqualität, Einstellung gegenüber PR und Marktgeschehen) systematisiert (s. Abb. 30). Die meisten Untersuchungen fokussierten auf den Krankenhausbereich, und der häufigste Endpunkt waren Befragungen und andere Studien zur Einstellung gegenüber Qualitätsinformationen, auf der Ebene der Patienten sogar die einzige Form. Leistungsanbieter (niedergelassene Ärzte, Krankenhäuser) lehnten PR größtenteils ab, Krankenkassen befürworteten sie, und bei den Patienten ergab sich ein widersprüchliches Bild mit der leichten Tendenz, dass bei sehr ausführlicher und technisch „gut gemachter“ Information über mehrere Informationskanäle sich die Einstellung gegenüber Krankenhäusern doch nachhaltig änderte. Diese Beobachtung wird durch neue Studien bestätigt

\section{PR: Akteure, Endpunkte}

\begin{tabular}{l|c|c|c|c} 
& Ergebnis-Q & Prozess-Q & Einstellung & Markt \\
\hline Patienten & & & $\downarrow \uparrow$ & \\
\hline niedergelassene Ärzte & $\varnothing$ & $\varnothing$ & $\downarrow$ & $\varnothing$ \\
\hline Krankenhäuser & $\uparrow$ & $\uparrow$ & $\downarrow$ & $+/-$ \\
\hline Kassen & $\varnothing$ & $+/-$ & $\uparrow$ & $\varnothing$ \\
\hline Gesundheitssystem & $\varnothing$ & $\varnothing$ & $\varnothing$ & $+/-$ \\
\hline
\end{tabular}

- nicht anwendbar $\varnothing$ keine Studien $\quad+$ /- indiff. Ergebnis $\quad \downarrow \uparrow$ pos./neg. Effekt

Abb. 30 Ergebnisse des Systematischen Reviews des Sachverständigenrates, bezogen auf die Akteure (horizontal) und die verschiedenen Endpunkte der Studien: Veränderungen bzgl. der Ergebnis- bzw. Prozessqualität, der Einstellungen (z.B. bei Befragungen) und im Marktgeschehen. PR = Public Reporting. (SVR 2008, Nr. 695ff) 
(Hibbard et al. 2012). Trotzdem gab es unter dem Strich keinen sicheren Hinweis darauf, dass Patienten als aktive „Marktteilnehmer“ die Qualitätsinformation aufnehmen und für eine Bevorteilung und Stärkung der „besseren“ Institutionen sorgen. Patienten sind zwar sehr an Qualitätsinformationen interessiert, machen sie jedoch nicht regelmäßig zur Grundlage ihrer Entscheidungen. Klar lässt sich jedoch herausarbeiten, dass Krankenhäuser selbst aktiv werden, wenn Public Reporting-Programme implementiert werden, da sie befürchten, in einer Ranking-Tabelle auf einem der letzten Plätze zu landen. Dieses Ergebnis korrespondiert gut mit dem Systematischen Review von Fung et al. (2008). In der Konsequenz ist Public Reporting besonders dort wirksam, wo bei Vorliegen einer hohen Krankenhausdichte der Konkurrenzdruck zwischen den Krankenhäusern besonders hoch ist (während Pay vor Performance unabhängig von diesem Konkurrenzdruck wirksam ist, s. Kap. 6.4).

Es gibt charakteristische und gut beschriebene Manipulationsmöglichkeiten, Daten können manipuliert werden (gaming), es kann eine aktive Risikoselektion (avoidance) betrieben und riskante Behandlungsmethoden können postponiert werden (withdrawal) (Green und Wintfeld 1995, Schneider und Epstein 1996, Scott und Ward 2006). Indirekt kann zu einer Indikationsausweitung kommen, da die Leistungsanbieter leichte Fälle präferieren (Werner und Asch 2005). Da Public Reporting kein Selbstläufer ist, muss eine sorgfältige Priorisierung der Ziele eines solchen Programmes geschehen (Meltzer und Chung 2014, Wright 2014).

\section{Zusammenfassung:}

Public Reporting ist besonders in Gebieten mit hoher Krankenhausdichte wirksam. Da Public Reporting Krankenhäuser über den befürchteten Reputationsverlust motiviert sind, liegt seine größte Wirksamkeit (im Gegensatz zu P4P) in Regionen mit hohem Konkurrenzdruck. Neben den bekannten Fehlanreizen wie gaming (Datenmanipulation), withdrawal (Verschiebung von komplikationsträchtigen Behandlungen) und avoidance (Risikoselektion) kann es zu einer Indikationsausweitung kommen (Attraktion leichter Fälle).

\subsection{Perspektive: Information der Patienten}

Bereits in Kapitel 2.4.7 wurde darauf eingegangen, dass in Zukunft Qualitätsindikatoren, die auf Patientenerfahrungen beruhen (sog. Patient-Reported Outcome Measures), eine größere Rolle spielen werden. Im Zusammenhang mit Public Reporting wurde das Konzept des selbständigen, voll informierten und aktiven Patienten ursprünglich ganz ins Zentrum der Überlegungen gestellt; er sollte entsprechend des Konzeptes des Qualitätswettbewerbes (s. Kap. 4.4) seinen Nutzen dadurch optimieren, dass er gemäß der in der Qualitätsberichterstattung enthaltenen Informationen die Einrichtung mit der besten Versorgung auswählt. Wie die in Kapitel 5.2 dargestellten Studien und Systemati- 
schen Reviews aber gezeigt haben, kann man das empirisch jedoch nicht nachvollziehen. Patienten sind sehr an Qualitätsinformationen interessiert (86\% wünschen Qualitätsinformationen [Geraedts und de Cruppé 2011]), aber für ihre Entscheidungen sind andere Faktoren ausschlaggebend. Offensichtlich spielt es eine große Rolle, inwieweit Patienten nicht nur kognitiv eine Information aufnehmen, sondern inwieweit sie diese mit einem Vertrauen in den Sachverhalt in Verbindung bringen, so wie es im persönlichen Kontakt (z.B. mit Familienangehörigen oder dem langjährig bekannten Hausarzt) der Fall ist.

Weiterhin ist zu beachten, dass nicht alle Patienten in gleicher Weise Informationen aktiv suchen und hierbei auch bereit sind, in einen Dissens mit ihrem Arzt bzw. Ärzten zu treten. In Abbildung 31 wird eine Typologie des Patientenverhaltens dargestellt, die hier hilfreich sein kann (Scheibler et al. 20o1). Patienten, die sich unabhängige Qualitätsinformationen besorgen und diese auch als entscheidungsrelevant ansehen, sind zweifelsfrei als aktive Patienten zu bezeichnen; dies heißt aber auch, dass passiv eingestellte Patienten hier bereits als Nutzer ausscheiden. Weiterhin verlangt die Entscheidung nach Qualitätsinformationen die Fähigkeit, sich kritisch distanzierend über einen Arzt bzw. eine Gesundheitseinrichtung kundig zu machen, mit der Option (sonst hat dieses Verhalten keinen Sinn), sich auch gegen den Arzt auszusprechen, falls die Qualitätsinformation nicht den Erwartungen entspricht. Aus dieser Sicht wäre also der nicht adhärente, intelligent-compliance Patiententyp (Quadrant rechts oben) der optimale Nutzer von Qualitätsinformationen. Allerdings wäre für den Fall, dass Informationen von einer als therapeutisch relevant angesehenen Institution mit hohem Vertrauensvorschuss angeboten werden, auch mit einem konsensbezogenen Verhalten im Sinne des Co-Therapeuten zu rechnen. Der Konsens würde sich hier nicht auf einen Arzt, sondern auf die Qualitätsinformation beziehen; es liegt auf der Hand, dass diese Option nur dann auftreten kann, wenn die Information optimal vermit-

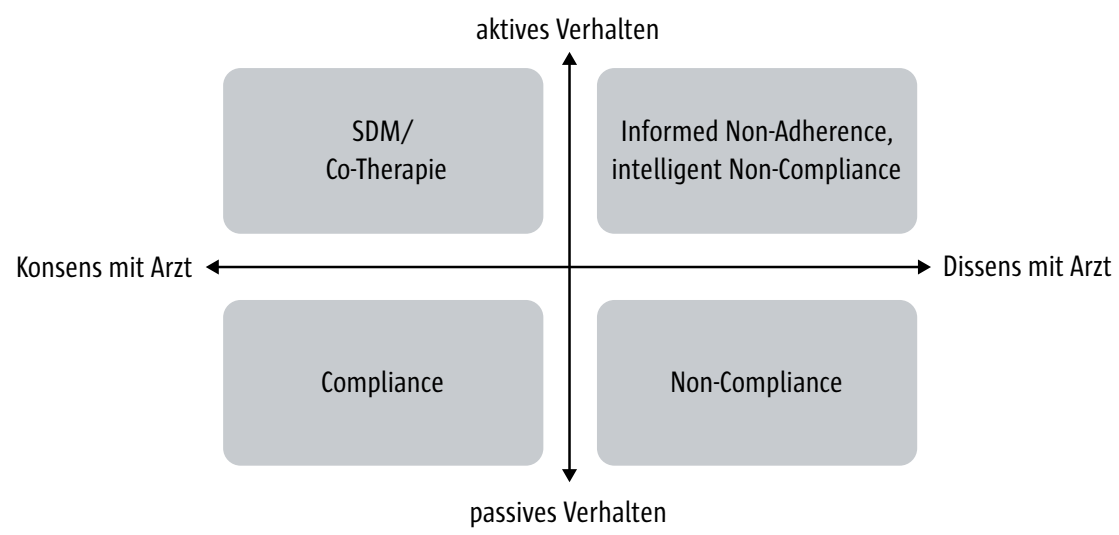

Abb. 31 Patiententypologie (n. Scheibler et al. 2001). SDM Shared Decision Making 
telt wird, eine Erklärung vielleicht für die Beobachtung aus einigen Studien, dass sehr intensiv und sehr vertrauenswürdig angebotene Information doch entscheidungsleitend sein kann (Hibbard et al. 2012).

Grundsätzlich kann nicht davon ausgegangen werden, dass Patienten bei allen Krankenhausbehandlungen die Institution frei wählen können (z.B. Notaufnahmen bei akuten Erkrankungen). Eine freie Wahl dürfte nur bei ca. 60\% aller Krankenhausbehandlungen möglich sein, ganz abgesehen davon, dass die gesetzlichen Bestimmungen nach $₫ 73$ Abs. 4 SGB V in Verbindung mit $₫ 92$ Abs. 1 Satz 2 Nr. 6 eine Wahl des nächstgelegenen Krankenhauses nahelegen (Geraedts u. de Cruppé 2011). Die Kriterien, nach denen Patienten sich entscheiden, sind auch in Deutschland relativ gut untersucht (Geraedts u. de Cruppé 2011). Es führen die Qualifikation der Ärzte, Sauberkeit und die Qualifikation der Pflege auf den ersten drei Plätzen, gefolgt von der Vorhaltung der besten Verfahren, Freundlichkeit, Einbeziehung, Spezialkompetenz, Zufriedenheit anderer Patienten, Komplikationen und Empfehlung durch Spezialisten. Weniger rationale Empfehlungen sind gefragt, sondern mehr individuell und emotional anschlussfähige, auf die Behandler bezogene Informationen, die Patienten aus dem ihren Netzwerken oder vom behandelten Arzt beziehen, und die als Basis für den Aufbau einer Vertrauensbeziehung gelten (Geraedts u. de Cruppé 2011). Als Quellen werden daher Verbraucherschutz oder Patientenverbände bevorzugt.

Es gibt auch Hinweise auf beunruhigende Approximationen, derer Patienten sich bedienen, z.B. die Annahme, dass höhere Preise höherer Qualität entsprechen (Carman et al. 2010). Weiterhin ist ein sozialer Gradient zu beobachten, im trade off zwischen Krankenhausnähe und Qualität ist ersterer Aspekt um so wichtiger, um so älter Patienten sind und eine um so niedrigere Ausbildung sie haben (Victoor et al. 2014).

\section{Zusammenfassung:}

Public Reporting setzt den „aktiven Patienten“ mit Mut zum Dissens voraus. Informationen zu Qualität sind sehr gefragt, Informationen von vertrauenswürdigen Institutionen (z.B. Verbraucherverbände) werden bevorzugt, die Hitliste wird von Qualifikation und Sauberkeit angeführt. Passive Patienten und Patienten mit hohem Alter sowie niedrigem Bildungsstand werden tendenziell benachteiligt. 